\title{
Comparing computed tomography (CT) angiography versus digital subtraction angiography for the screening of traumatic pseudoaneurysms in transcranial stab injuries
}

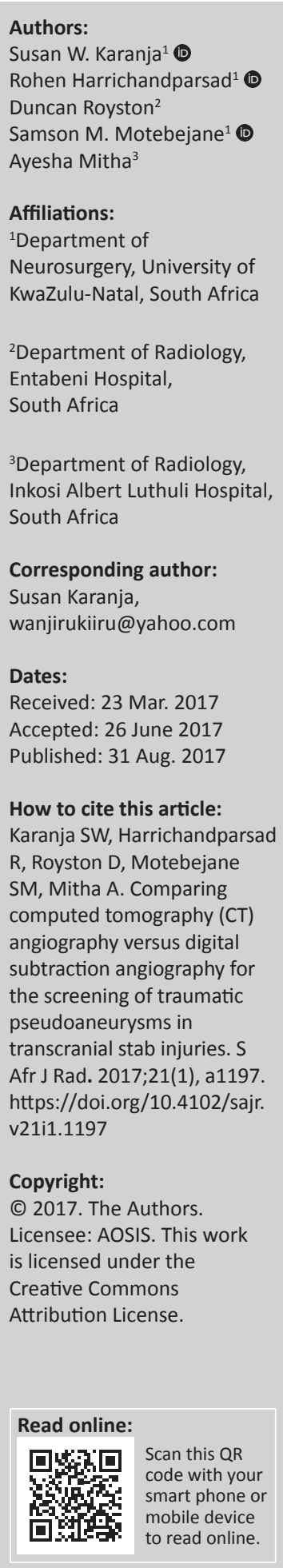

Introduction: Transcranial stab injuries among the civilian population in South Africa are more common than in the West. In a study conducted in Durban, South Africa, in 1992, transcranial stab injuries accounted for $6 \%$ of all head injuries admitted to the neurosurgical unit. Digital subtraction angiography (DSA) has been the gold standard for diagnosing traumatic pseudoaneurysms. Its use as a screening tool is, however, limited and carries risks of neurological deficits. We postulate that the newer generation computed tomography (CT) scanner would serve as a better screening tool for traumatic pseudoaneurysms following transcranial stab injuries with the blade removed, provided the image quality is good.

Methods: All patients admitted with a stab to the head with total calvarial penetration from September 2014 to January 2016 were included in the study. Those with a retained metallic fragment, incomplete imaging protocols, no penetration of the blade into the brain parenchyma or other causes of penetrating head injury were not included in the study. A retrospective chart review was then done on a prospectively recruited patient cohort. All CT angiograms were performed using a dual source CT scanner.

Results: A total of 26 patients met the inclusion criteria. There were seven vascular injuries identified on DSA: three traumatic pseudoaneurysms and four vessel cut-offs. One traumatic pseudoaneurysm was missed on computed tomography angiography (CTA) because of a poor quality scan. The average sensitivity and specificity of CTA compared with DSA was $67 \%$ and 95.5\%, respectively. Negative predictive value for CTA was $99.5 \%$.

Conclusion: A good quality CTA carried out on the newer generation CT scanners allows for use of CTA as a screening tool for patients with a transcranial stab injury and no retained blade or metallic foreign bodies.

\section{Introduction}

Transcranial stab injuries among the civilian population in South Africa are more common than in the West. Penetrating head injury (PHI), which includes both missile injuries and transcranial stab injuries, has been reported as representing about $0.4 \%-5 \%$ of all head injuries in the West. ${ }^{1,2}$ In a study conducted in Durban, South Africa, in 1992, transcranial stab injuries alone represented about $6 \%$ of all head injuries admitted to the neurosurgical unit. Most patients present with the weapon already removed by the assailant. ${ }^{3}$ Non-contrast computed tomography (CT) brains typically show a slot fracture with an underlying tract haematoma (Figure 1). Vascular complications of PHI are reported in about $5 \%-40 \%$ of these cases. ${ }^{1}$ Du Trevou et al. reported vascular injuries in $12 \%$ of patients with transcranial stab injuries and this study served to highlight the importance of early digital subtraction angiography (DSA) in patients with PHI for detection of vascular injuries. DSA following PHI is the gold standard for diagnosis of vascular injury. ${ }^{3}$

The role of computed tomography angiography (CTA) in the setting of PHI is currently undefined because of a paucity of literature. The sensitivity of older generation CTA was deemed inadequate for screening of traumatic pseudoaneurysms as these are typically peripherally located lesions affecting smaller sized vessels as compared to the circle of Willis (COW). However, with the newer generation CT scanners, we postulated that this would serve as a better screening tool than DSA.

Factors that limit the use of DSA as a screening tool in the acute setting include that it is labour intensive, requires skilled personnel, is expensive and is not readily available $24 \mathrm{~h}$ a day. 

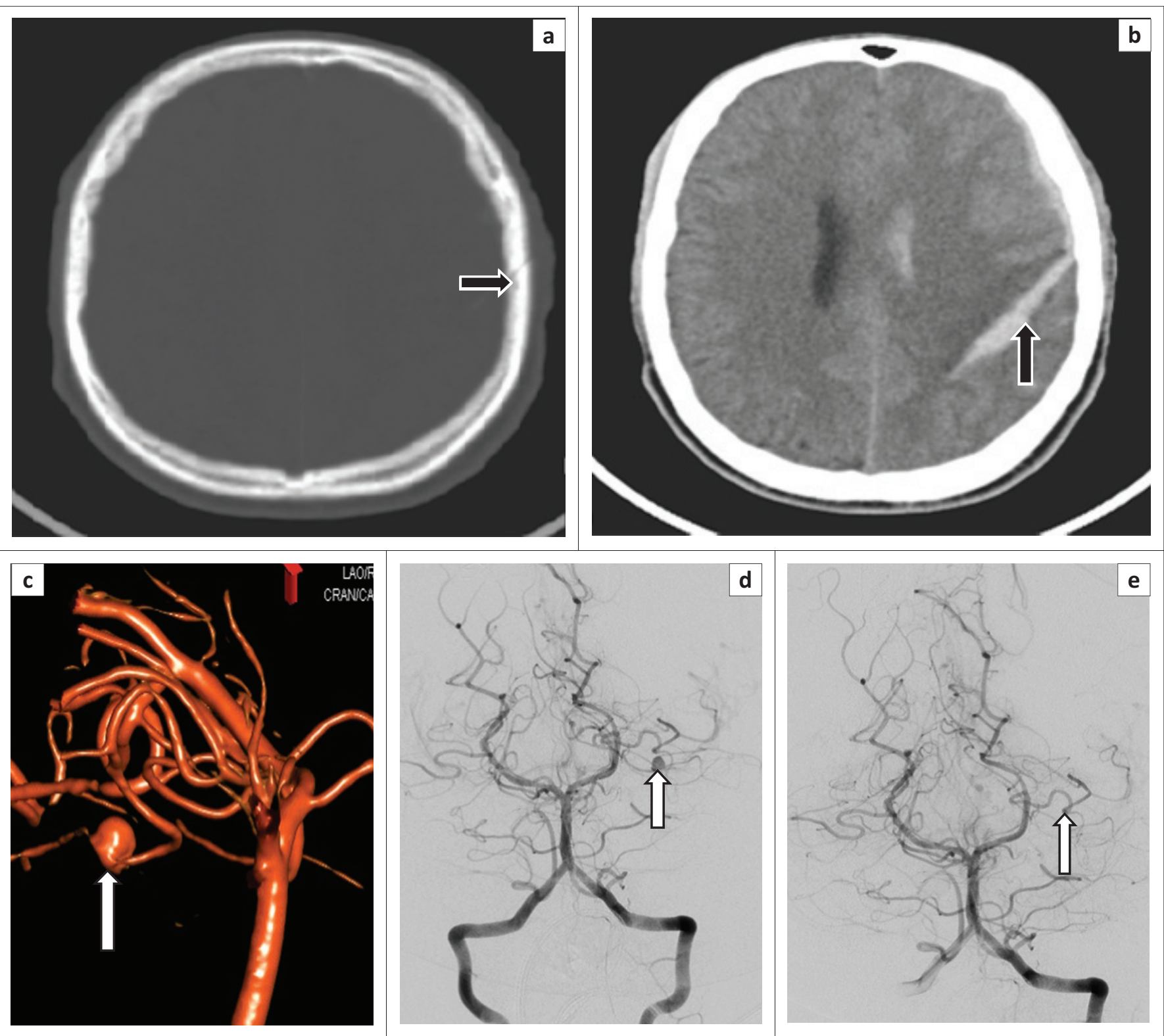

FIGURE 1: (a) Computed tomography (CT) scan (bone window) showing a slot fracture with complete calvarial penetration, (b) CT scan of the brain showing a tract haematoma, (c) Three-dimensional reconstruction from the digital subtraction angiography (DSA) showing a traumatic pseudoaneurysm, (d) DSA showing a traumatic pseudoaneurysm before endovascular intervention and (e) DSA done following parent vessel occlusion showing complete exclusion of the aneurysm.

Furthermore, DSA is invasive and carries a $0.4 \%-12.2 \%$ risk of neurological deficit which is permanent in up to $5.4 \%$ of cases. ${ }^{4}$ CTA would act as a suitable screening tool because of its widespread availability, rapid acquisition times, noninvasive nature and 24-h availability of personnel. It would thus form part of the initial trauma imaging and would allow triage decisions to be made rapidly and facilitate early intervention. Early diagnosis of vascular injuries in PHI would aid in preventing secondary brain injury through haemorrhage or infarction.

CTA as a screening tool has been shown to have a reduced sensitivity and specificity in the presence of metallic artefacts, which would be the case with a retained knife blade. In this setting, DSA is still the preferred modality for detecting associated vascular injuries. This sensitivity was noted to be lower for non-Traumatic Intracerebral Aneurysms (nTICA) injuries of the second and third order branches of the intracranial vessels. ${ }^{5}$ The objective of this study was to determine the sensitivity of 128-slice dual source CT angiography as compared with DSA as a screening tool for intracranial vascular injuries in patients presenting with transcranial stab injuries and no retained blade. The study received ethics approval from the University of KwaZulu-Natal Biomedical Ethics Committee (reference number BE038/16).

\section{Methods}

The study was carried out at a university teaching hospital in Durban, South Africa. A prospectively collected cohort of consecutive patients admitted with a stab to the head with total calvarial penetration and no retained blade from September 2014 to January 2016 was retrospectively reviewed in this study. Both CTA and DSA were performed 
during admission. Patients who had incomplete imaging protocols or other causes of PHI like missile or machete injuries were excluded. Those found with a retained knife blade or no penetration of the brain parenchyma were also excluded from the study.

\section{Imaging protocol}

All patients in the study were required to have informed consent for both CTA and DSA. This was obtained from the patient or next of kin. All patients had an initial non-contrast CT brain scan performed followed by $\mathrm{CT}$ angiography which was done according to the protocol set by the institution's radiology department (Table 1). CT angiography at the institution was done on the Siemens Definition Flash Dual Source Scanner (Siemens AG, Wittelsbacherplatz, Germany).

A team comprising fellowship trained neurosurgeons performed the DSA studies on the next available elective day after the CTA. DSA was performed using the Siemens Artis Zee Biplane Machine (Siemens AG, Wittelsbacherplatz, Germany). A standard Seldinger technique was used. A carotid run on the side of injury was done. A vertebral run was also performed if the injury was more posteriorly located.

\section{Image interpretation}

Two independent neuroradiologists reviewed both the CT angiography and DSA images (D.R. and A.M.). The source images were not available for review but the $3 \mathrm{D}$ reconstructions were available for review. Findings by the two radiologists were correlated with the initial reports which had been done using source images. The radiologists were blinded to the findings on unenhanced CT brain and the reports of the CT angiography. The reviewers graded the quality of CT angiography using the criteria in Table 2. They assessed for vasospasm and also identified any vascular injuries on CTA and DSA. We were particularly interested in identifying traumatic pseudoaneurysms which are lesions requiring treatment.

\section{Statistical analysis}

Analysis techniques included descriptive statistics. Means and frequencies were utilised to explore the data. Contingency tables were utilised to calculate the sensitivity, specificity, negative predictive value (NPV) and positive predictive

TABLE 1: Protocol used for conducting computed tomography angiography and image reconstruction

\begin{tabular}{ll}
\hline Protocol & Instructions for use \\
\hline Contrast Administered & $50 \mathrm{mls}$ at a minimum of $5 \mathrm{mLs} / \mathrm{sec}$ via injector \\
Exposure & Up to the level of the ascending aorta \\
Pre-contrast head scan & Always ensure that the scan delay is 2 seconds or less \\
Head Angiogram & Must match the pre-contrast CT brain \\
Reconstruction & Pre-contrast scan: \\
& $\begin{array}{l}\text { Reconstruction type: Axial } \\
\text { Slice: } 0.6 \times 0.4 \mathrm{~mm}\end{array}$ \\
& Head angiography reconstruction: \\
& Slice: $0.6 \times 0.4 \mathrm{~mm}$ \\
& 2mm axials, coronal and sagittal and \\
& subtracted images \\
\hline
\end{tabular}

$\mathrm{mLs}$, millilitres; $\mathrm{mLs} / \mathrm{sec}$, millilitres per second; $\mathrm{CT}$, computed tomography values (PPV) for each observer. The means of these values were then obtained.

\section{Results}

A total of 26 patients met the inclusion criteria for the study. Of the 26 patients, 25 were males and 1 was female. The mean age of patients in the study was 26.23 years \pm 7.32 . The mean Glasgow Coma Scale (GCS) on presentation was $13 \pm 2$ with 20 patients presenting with left-sided stabs and 6 with rightsided stabs; the findings are summarised in Table 3 . Figure 2 shows the common regions of the stab wounds in the patient population. The average duration of time between CTA and DSA was 6.38 days \pm 5.85 .

The first analysis consisted of comparing the radiologist CTA report to the neurosurgical DSA findings. Vascular injuries were found in 10 patients on CTA and in 7 patients on DSA. Four patients had a false-positive CTA report and a vascular injury was missed in one patient who had a traumatic pseudoaneurysm on DSA. This gave a sensitivity of $67 \%$ and specificity of 91\% for CTA (PPV 50\% and NPV 95\%) when compared with DSA. Of the seven vascular injuries reported, three were traumatic pseudoaneurysms which warranted endovascular treatment. The remaining four patients had vessel cut-off on DSA. Figure 3 summarises the distribution of patients with vascular injuries and shows those missed on CTA.

The second analysis was then performed by two radiology consultants (D.R. and A.M.) who compared CTA and DSA for detection of traumatic pseudoaneurysms. On CTA review, each of the radiologists missed one traumatic pseudoaneurysm but they identified all three on DSA. The CTA of the missed injury was noted as being of poor quality. From the data, A.M. had a CTA sensitivity of $67 \%$ and specificity of $100 \%$ (PPV $=$ $100 \%, \mathrm{NPV}=96 \%$ ). D.R. had a CTA sensitivity of $67 \%$ and specificity of $91 \%(\mathrm{PPV}=50 \%, \mathrm{NPV}=95 \%)$. This gave anaverage sensitivity of $67 \%$ and specificity of $95.5 \%$ $(\mathrm{PPV}=75 \%, \mathrm{NPV}=99.5 \%)$ for CTA when compared with DSA.

\section{Discussion}

Complications of transcranial stab injuries can be vascular or infective with vascular injury being either acute or

TABLE 2: Computed tomography angiography quality grading criteria used by both radiologists.

\begin{tabular}{ll}
\hline CTA quality & Findings \\
\hline Good & Good arterial opacification, no venous overlap \\
Moderate & Good arterial opacification, some venous overlap \\
Poor & Poor arterial opacification with venous overlap \\
\hline
\end{tabular}

CTA, computed tomography angiography.

TABLE 3: Summary of data from patient cohort.

\begin{tabular}{llll}
\hline Patient demographic & Mean & SD & Range \\
\hline Age (years) & 26.25 & \pm 7.32 & $17-45$ \\
GCS at presentation & 13 & \pm 2.14 & $8-15$ \\
Days between CTA and DSA & 6.38 & \pm 5.85 & $-1-23$ \\
\hline
\end{tabular}

CTA, computed tomography angiography; DSA, digital subtraction angiography; GCS, Glasgow Coma Scale.

$N=26$ (with 25 male patients and 1 female patient). 


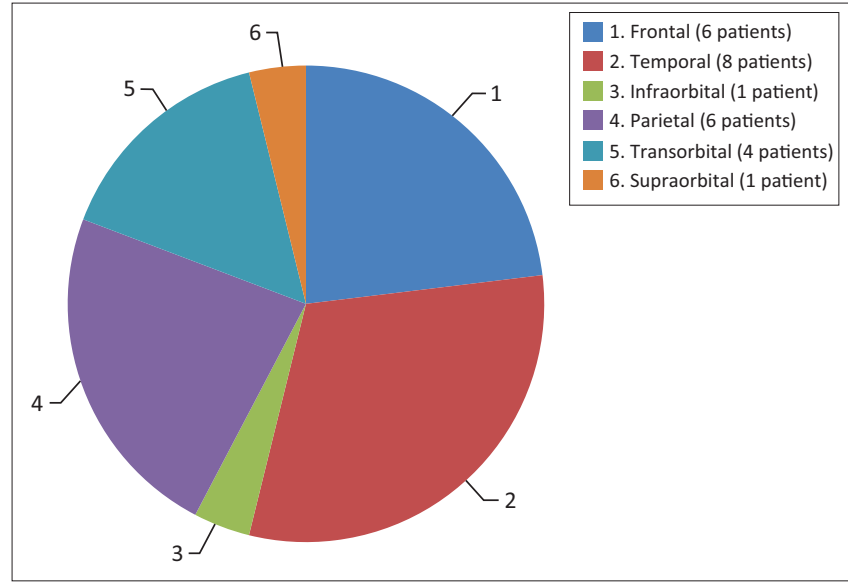

FIGURE 2: Number of patients and region of stab wounds.

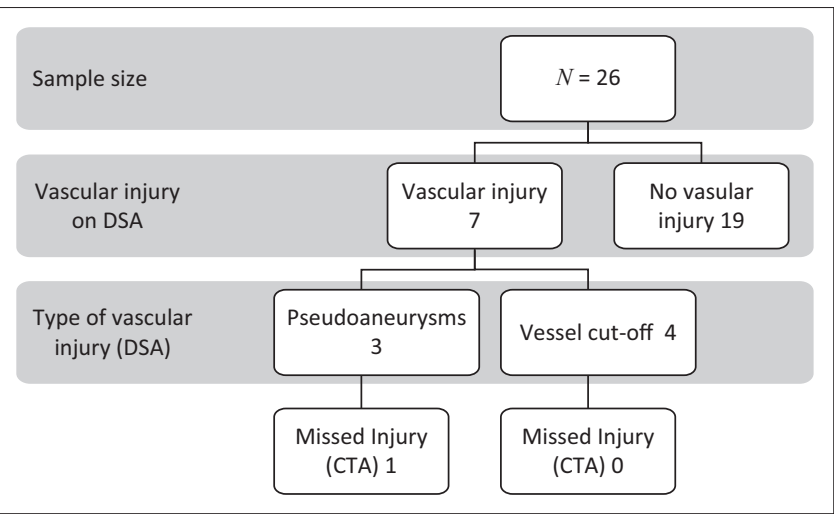

FIGURE 3: Flow-chart summarising patient distribution with regards to diagnosis of vascular injury by digital subtraction angiography.

delayed. ${ }^{6}$ We focused on acute vascular injury following transcranial stab injury with the blade removed and evidence of intracranial penetration. From the results obtained, the focus was on traumatic pseudoaneurysms which though accounting for approximately $1 \%$ of intracranial aneurysms have morbidity and mortality rates as high as $50 \%$ if left untreated because of the high risk of rupture. ${ }^{78}$ Early detection and treatment of traumatic pseudoaneurysms would thus prevent secondary injury through haemorrhage. ${ }^{9}$ A $12 \%$ incidence of traumatic pseudoaneurysms following stab head has previously been reported in our department. The same study also showed a mortality rate of $26 \%$ for patients who presented with the blade removed compared with $11 \%$ for those with the blade in place. ${ }^{3}$

Bodanapaly et al. sought to determine the feasibility of CTA for detecting vascular injury following PHI. They assessed both missile and non-missile injuries and had a CTA sensitivity rate of $72.7 \%$ for all vascular injuries, which was lower than the $98 \%$ reported for intracranial saccular aneurysms. ${ }^{10}$ In our study, the sensitivity was $67 \%$ for traumatic pseudoaneurysms on review of imaging by senior radiologists. In this study, three pseudoaneurysms were identified on DSA and subsequently underwent endovascular parent vessel occlusion. On CTA, both radiologists missed one of these injuries. This could be accounted for by the following factors: (1) No source images were available for review by radiologists, (2) lack of admission non-contrast CT scan to allow identification of site of injury and (3) CTA was noted to be of poor quality.

The average NPV for the two senior radiologists even without the source images was 99.5\%. Excluding the missed pseudoaneurysm because of poor quality CTA, the sensitivity of CTA for detecting traumatic pseudoaneurysms would be $100 \%$. This shows that CT angiography would be appropriate for screening patients with transcranial stab injuries and no retained metallic foreign bodies. CTA is non-invasive, relatively inexpensive, readily available and can be performed immediately after routine unenhanced CT scanning. In view of the advantages offered by a good quality CTA, it is our recommendation that CTA be used as a screening tool for the detection of traumatic pseudoaneurysms following PHI. A senior radiologist or neuroradiologist should review the images, in conjunction with the non-contrast CT brain to focus on the area of potential injury. Should they find the image quality to be poor, a repeat CTA or DSA would be the next step. DSA is indicated in patients with retained metallic foreign body, poor quality CTA or a CTA where a vascular injury cannot be confidently excluded. Should vasospasm be found on CTA our recommendation is to repeat the CTA after 1 week, so as not to miss any injuries. Following identification of a traumatic pseudoaneurysm in our centre, all patients were treated by endovascular parent vessel occlusion, but surgical parent vessel occlusion is an alternative in centres with no endovascular option.

DSA remains the gold standard for diagnosis of vascular injuries following PHI. In our centre, it remains the main mode of investigating patients with transcranial stab injuries for vascular lesions. The current role of CTA following PHI is as one of the main screening tools for vascular injuries. Both CTA and DSA are used interchangeably following PHI. ${ }^{11}$ DSA is reserved for select cases or where intervention is likely to be needed. Like with DSA, it is recommended to repeat the CTA to avoid missing delayed vascular injuries. The disadvantages of CTA in the setting of transcranial stab injuries are potential artefacts affecting image quality and the inability to have therapeutic intervention in the same setting. ${ }^{12}$

\section{Study limitations}

This study is limited by the small sample size, the subjective grading of CTA by the radiologists and a lack of source imaging for review by the radiology consultants. However, despite not having source images available, all traumatic pseudoaneurysms were identified by both radiologists on good quality CTA. The delay of up to 23 days between DSA and CTA is also a limitation as during this time any vasospasm that may have been present would have resolved, unmasking any injuries that would have been missed by CTA. This would introduce a bias, making DSA seem more sensitive than CTA. 


\section{Conclusion}

A good quality CTA on the newer generation CT scanners is an effective screening tool for patients presenting with a transcranial stab injury and no retained blade or metallic foreign objects. It provides a rapid and more cost-effective means of triaging these patients. This allows for earlier intervention in patients with traumatic pseudoaneurysms, thus reducing the morbidity and mortality associated with these rare but dangerous lesions. DSA is indicated if the CTA is of poor quality and a traumatic pseudoaneurysm cannot be confidently excluded, or if there is a retained metallic foreign body.

\section{Acknowledgements}

We would like to acknowledge Yusentha Balakrishna, South African Research Council, for her role in the statistical planning and analysis of this article.

\section{Competing interests}

There are no competing interests to declare.

\section{Authors' contributions}

S.W.K. was involved in conceptualisation of the article, collection of data, preparation of the manuscript and preparation of the data collection questionnaires. R.H. was involved in approving the concept of the article, review of the manuscript and approval of the final draft. D.R. was involved in data collection and interpretation and approval of the final copy of the manuscript. A.M. was involved in data collection and interpretation. S.M.M. was involved in conceptualisation of the article and approval of the final copy.

\section{References}

1. Bieler D, Franke AF, Hentsch S, et al. Gunshot and stab wounds in Germanyepidemiology and outcome: Analysis from the TraumaRegister DGU $^{\circledast}$. Unfallchirurg. 2014;117:995-1004. https://doi.org/10.1007/s00113014-2647-7

2. Bodanapally UK, Saksobhavivat N, Shanmuganathan K, Aarabi B, Roy AK. Arterial injuries after penetrating brain injury in civilians: Risk factors on admission head computed tomography. J Neurosurg. 2015;122:219-226. https://doi. org/10.3171/2014.9.JNS14679

3. du Trevou MD, van Dellen JR. Penetrating stab wounds to the brain: The timing of angiography in patients presenting with the weapon already removed. Neurosurgery. 1992;31:905-911;discussion 11-12. https://doi.org/10.1227/ 00006123-199211000-00012

4. Stallmeyer MJ, Morales RE, Flanders AE. Imaging of traumatic neurovascular injury. Radiolo Clin North Am. 2006;44:13-39.

5. Nathoo N, Boodhoo H, Nadvi SS, Naidoo S, Gouws E. Transcranial brainstem stab injuries: A retrospective analysis of 17 patients. Neurosurgery. 2000;47: 1117-1122. https://doi.org/10.1097/00006123-200011000-00018

6. Taylor AJ, Peter JC. Patients with retained transcranial knife blades: A high risk group. J Neurosurg.1997;87:512-515. https://doi.org/10.3171/jns.1997. 87.4.0512

7. Larson PS, Reisner A, Morassutti DJ, Abdulhadi B, Harping JE. Traumatic aneurysms. Neurosurg Focus. 2000;8:Article 4.

8. Neuroimaging in the management of penetrating brain injury. J Trauma. 2001;51(2 Suppl):S7-S11.

9. Bodanapally UK, Krejza J, Saksobhavivat N, et al. Predicting arterial injuries after penetrating brain trauma based on scoring signs from emergency CT studies. Neuroradiol J. 2014;27:138-145.

10. Bodanapally UK, Shanmuganathan K, Boscak AR, et al. Vascular complications of penetrating brain injury: Comparison of helical $\mathrm{CT}$ angiography and conventional angiography. J Neurosurg. 2014;121:1275-1283.

11. Syed FK, Muhammad SS, Muhammad ZT, Syed AE, Shahan W. Management of penetrating head injury. J Emerg Trauma Shock. 2011:395-402.

12. Nikki T, Cortny D, Amanda S, Warren R. Neuroimaging in adult penetrating brain injury: A guide for radiographers. J Med Radiat Sci. 2015;62:122-131. 